\title{
CHOICE REACTION TIME FOR REPETITIONS AND NON-REPETITIONS
}

\section{A RE-EXAMINATION OF THE INFORMATION HYPOTHESIS ${ }^{1}$}

\author{
S. KORNBLUM ${ }^{2}$ \\ Mental Health Research Institute, University of Michigan, Ann Arbor, USA
}

\begin{abstract}
The principal objective of this study was to describe some of the characteristics of the responses to repetitions and alternations in choice RT tasks. To the extent that these responses were shown to be differentially sensitive components of the overall RT, whose weighted sum yielded the linear relationship between the mean overall R.T and ransmitted information, it was suggested that a more detailed study of these components may be the move fruitful approach toward understanding the microstructure of RT processes. Clearly these arguments are not restricted to RT tasks alone but are applicable to a wide variety of experimental results which have been described in terms of a linear relationship between information and performance measures. Our results in no way deny the descriptive statements of the information hypothesis. On the contrary, our data in fact provide one more confirmation of the hypothesis. However, we have pointed toward a possible artifactual reason for the relationship and a change of emphasis has been suggested which may lead to more explacatory types of staternents than have been forthcoming from the information approach.
\end{abstract}

\section{INTRODUCTION}

Five years ago Bertelson published the first of several studies (Bertelson, $1961,1963,1965)$ in which the reaction time to conditional events was examined in an experimental situation which was explicitly designed to separate the repetition from the non-repetition components in a choice reaction time task. Brieffy, he found that the reaction time for a repeated event was different than for a non-reptated event, the latter being the slower. These results provided

1 This wark was partially supported by NIH grant MH-07197 to the author. One part of these data was presented at the Xviii International Congress of Psycholczy, Moscow, 1966. A more detailed and fuller account of the main experiment will appcar elsewhere.

2 For their help on various phases of this project I wish to thank Lois Ball, Judy Bergess, Robert Fisieh and Sally Wacker. 
experimental verification for what had previously only been anecd otal reports of incidental observations (HYMAN, 1953; WELFORD, 1959).

Bertelson's findings are the first of three points which I should like to bring together and consider as a new and perhaps more profitable context in which to examine choice reaction time processes.

The second of these points is simply a reminder of the shape of the function relating the information measure $\left(H^{H}\right)$ and the probability of alternation $\left(p_{\mathrm{a}}\right)$ in sequences where the signals are equiprobable. Given a fixed number of alternatives $(K)$ and equiprobable signals $\left(p_{i}=1 / K\right)$ the average uncertainty of the sequence increases as a function of $p_{3}$ to a maximum where $p_{a}=(K-1) / K$. That is, $H$ and $p_{a}$ are highly correlated over a large range of $p_{a}$ values.

The third and final point is also in the form of a reminder and has to do with a very simple property of the overall mean of a RT distribution. Since separating the repetitions from the non-repetitions, or alternations, partitions the data, the overall mean $(\bar{R} \bar{T})$ is the sum of the means for repetitions $\left(\bar{R} \bar{T}_{\mathrm{r}}\right)$ and alternations $\left(\bar{R}_{\mathrm{T}}\right)$ each weighted by their own probability of occurrence $\left(p_{r}\right.$, and $\left.p_{a}\right)$; i.e.

$$
\bar{R} \bar{T}=p_{\mathrm{r}} \bar{R} \bar{T}_{\mathrm{r}}+p_{\mathrm{a}} \bar{R} \bar{T} \mathrm{a} .
$$

From these three points at least two rather compelling arguments emerge.

\subsection{The confounding argument}

While a great many studies have been done which indicate that $\bar{R} \bar{T}$ increases linearly with $H t$ the bulk of the evidence rests on sequences in which the signals were equiprobable. But we have just pointed out that unless a special sampling technique is used for such sequences $H$ is monotone in $p_{a}$ up to $(K-1) / K$. Therefore, to attribute the rise in $\bar{R} \bar{T}$ to $H$, as the information hypothesis does is, of course, to ignore this serious confounding between $H$ and $p_{a}$. This confounding is present on logical grounds alone. And, while it is obvious in the equiprobable case, it is also difficult to avoid in the nonequiprobable case. A more appropriate test of the information hypothesis would consist of using sequences in which $p_{a}$ and $H$ are either uncorrelated, or negatively correlated.

\subsection{The artifactual argument}

Given that $\bar{R} T_{\mathrm{B}}>\bar{R} \bar{T}_{\mathrm{r}}$ and that for a given $K$ an increase in $H$ is obtained by increasing $p_{k}$, the observed net increase in $\bar{R} \bar{T}$ may simply be attributable to the shifts in the proportion of slower and faster responses in the overall $\bar{\Sigma} \bar{T}$ distribution. For example-in an equiprobable eight choice task with maximum uncertainty, 7/8 of all responses are alten dtion responses. By fixing $p_{a}$ at .5 and keeping the signals equiprobable, $H$ is reduced to 2.4 bits and according to the information hypothesis one would expet a decrease in $\bar{R} \bar{T}$. However, that decrease may simply be due to the fact that the proportion of 
alternations has been reduced from $7 / 8$ to $1 / 2$ with a corresponding increase in the repetitions from $1 / 8$ to $1 / 2$. Since the alternations are slower than the repetitions, it is reasonable that this shift in their relative weightings should cause a decrease in $\bar{R} \bar{T}$.

Repetitions and alternations are thus components of the overall RT distribution whose relative weightings have been changed with the conditions in such a way as to make the net changes in the $\bar{R} \bar{T}$ consistent with the changes that one would expect under the information hypothesis. However, we know very little of these components. Indeed, a vast range of functions may be envisaged for $R T_{\mathrm{a}}$ and $R T_{\mathrm{r}}$ whose weighted sum would yield the linear reGationship described by the information hypothesis. The principal object of the present study is to examine some of these properties of repetitions and alternations as the first istep in an atiempt to understand the microstructure of choice RT processes.

\section{Method}

\subsection{Equipment}

The control and recording portions of the equipment were located in a room shich was separate from the one in which the subject sat. Control and recording was Jone by means of punched paper tape which was read at $300 \mathrm{cps}$ and punched at $60 \mathrm{cps}$. The output tape contained all the trial specifications, the subject's RT in milliseconds, the specific response that he made and whether or not it was correct. The gating and circuitry which constitute the heart of the equipment have been deseribed elsewhere (Peretz and Kornslum, 1966).

The subject sat in an adjoining room $51 / 2 \mathrm{ft}$ from a vertical board on which the signatis were presented. The signals themselves consisted of eight neon lights mounted in two slight ares to indicate each hand. The responses consisted of pressing the appropriate one of $K$ keys which required 5-6 ounces pressure and $1 / 8$ " travel to be depressed.

\subsection{Experimental conditions}

This was a serial task in which a 137 msec delay was used between a subject's response and the appearance of the next signal.

Sequences with two, four, and eight alternatives and equiprobable signais were constructed, each with probability of alternation of $1 / 2,3 / 4$, and $7 / 8$. In addition to these nine sequences two more were constructed: an eight-choice sequence with an average unicertainty of 2.00 ) bits and a four-shoice sequence with an average uncertainty of 1.00 bit (see tatile 1 ).

Twelve right-handed males participated as subjects in the study and were run for three days in addition to one day of training. Each subject was run on eight conditions per day with 400 trials per sondition. Each series of 400 trials was divided into quarters, and medians were taken for each quarter for the repetitions, alternations and overall RT. Means of these medians over quarters were then taken for the three types of responses. 


\section{TABLE 1}

This table illustrates the eleven experimental conditions of the main experiment: $\boldsymbol{K}$ denotes the number of alternatives in the secuence; $p_{a}$ denotes the probability of alternation. The cell entries are the average stimulus uncertainty values.

\begin{tabular}{|c|c|c|c|c|c|}
\hline $\begin{array}{c}p_{\mathbf{a}} \rightarrow \\
K \\
+\end{array}$ & $1 / 2$ & $3 / 4$ & $7 / 8$ & $1 / 5$ & $3 / 8$ \\
\hline 2 & 1.00 & .81 & .54 & \multirow{3}{*}{1.00} & \multirow[b]{3}{*}{2.00} \\
\hline 4 & 1.79 & 2.00 & 1.93 & & \\
\hline 8 & 2.4 & 2.91 & 3.00 & & \\
\hline
\end{tabular}

\section{RESULTS AND DISCUSSION}

The results for the overall mean RT are fairly straightforward. First, a practice effect is evident which accounts for a $33 \mathrm{msec}$ average improvement from day 1 to day 3. This improvement is larger the greater the number of alternatives so that practice would also have the effect of reducing the slope of the line. Point for point, however, the shape of most of the functions is almost identical for all three days, my discussion will therefore be restricted to the results of the third day. Secondly, a straight line drawn by eye appears to fit the overall mean RT for the third day reasonably well, which, of course, is in accordance with the information hypothesis (see fig. 1). Our data, therefore, conform quite clearly to that vast body of evidence in which performance measures have been shown to vary linearly with transmitted information.

Since stimulus uncertainty is as convenient a metric as any to use under some circumstances, let us next look at $\bar{R} \bar{T}_{\mathrm{r}}$ as a function of $H$. Except for the two-choice case $\bar{R} \bar{T}_{\mathrm{r}}<\bar{R} \bar{T}$ (see fig. 1). And this should not be surprising for it merely corroborates Bertelson's indings in more detail. $\bar{R} \bar{T}_{5}$ also increases as a joint function of $K$ and $p_{\mathrm{a}}$. Thus, the 1.93 bit condition has the highest $p_{\mathrm{a}}$ value, as well as the longest $\bar{R} \bar{T}_{\mathrm{r}}$ of the four choice conditions. This increase in $\bar{R} \bar{T}_{\mathrm{r}}$ with increasing values of $p_{\mathrm{a}}$ is an instarice of the commonly reported finding of $\bar{R} \bar{T}$ being an inverse function of the probability of the stimulus event, in this particular case the stimulus event being a repetition.

This finding is in sharp contrast to $\bar{R} \bar{T}_{\mathrm{a}}$. Unlike $\bar{R} \bar{T}_{\mathrm{r}}, \bar{R} \bar{T}_{\mathrm{a}}$ is relatively constant for a given $K$ irrespective of changes in $p_{a}$, and drops precipitously as $K$ is reduced. Thus, with the exception of the two-choice case again, $\bar{R} \bar{T}_{\mathbf{a}}$ appears to be relatively insensitive to changes in $p_{\mathrm{a}}$.

In every instance thus far the results for the two-choice conditions have stood out as exceptions from the rest of the data. In one instance these exceptions may be reconciled, in the other they cannot. Let us first consider the reconcilable case in which $\bar{R} \bar{T}_{\mathrm{r}}$ decrezses while $\bar{R} \bar{T}_{\mathrm{a}}$ increases as a function 
of $H$. In the four and eight-choice conditions not only does $\bar{R} T_{\mathrm{a}}$ remain fairly con:tant over changes in $H$, but $\bar{R} \bar{T}_{\mathrm{r}}$ increases as a function of $H$. This is true in all crives but one-the four-choice condition in which $\bar{R} \bar{T}_{\mathrm{r}}$ is longer for 1.93 bits than it is for 2.00 bits, and this has already been accounted for. In the kwo-choice conditions the decrease in $\boldsymbol{H}$ was always achieved by increasing $D_{2}$. Since we have already established that $\bar{R} \bar{T}_{\mathrm{r}}$ is inversely related to $p_{\mathrm{r}}$ in the four and eight-choice conditions, the two-choice repetition data are perfectly consistent with these findings. What remains to be accounted for is the fact that for two-choice, not only are the repetitions sensitive to $p_{r}$ but the alternations appear to be equaliy sensitive to it. In this respect, the two-choice condition would almosi appear to be a special case of choice behavior-as I believe it is.

Le me, therefore, digress for a moment and report some prelirainary resulis of an experiment which we are currently conducting with a two-shoice serial iask. The interval that was used between a response and the apperrance of the next signal was $270 \mathrm{msec}$, and this may be important for some of the results that were obtained. The object of this experiment is to asseis the relative contribution of the albsolute and conditional probabilities of a signal, as well as the average stimulus uncertainty, to $\bar{R} \bar{T}_{\mathrm{r}}$ and $\bar{R} \bar{T}_{\mathrm{b}}$. The resulting three-dimensional space of independent variables may be sliced in a variety

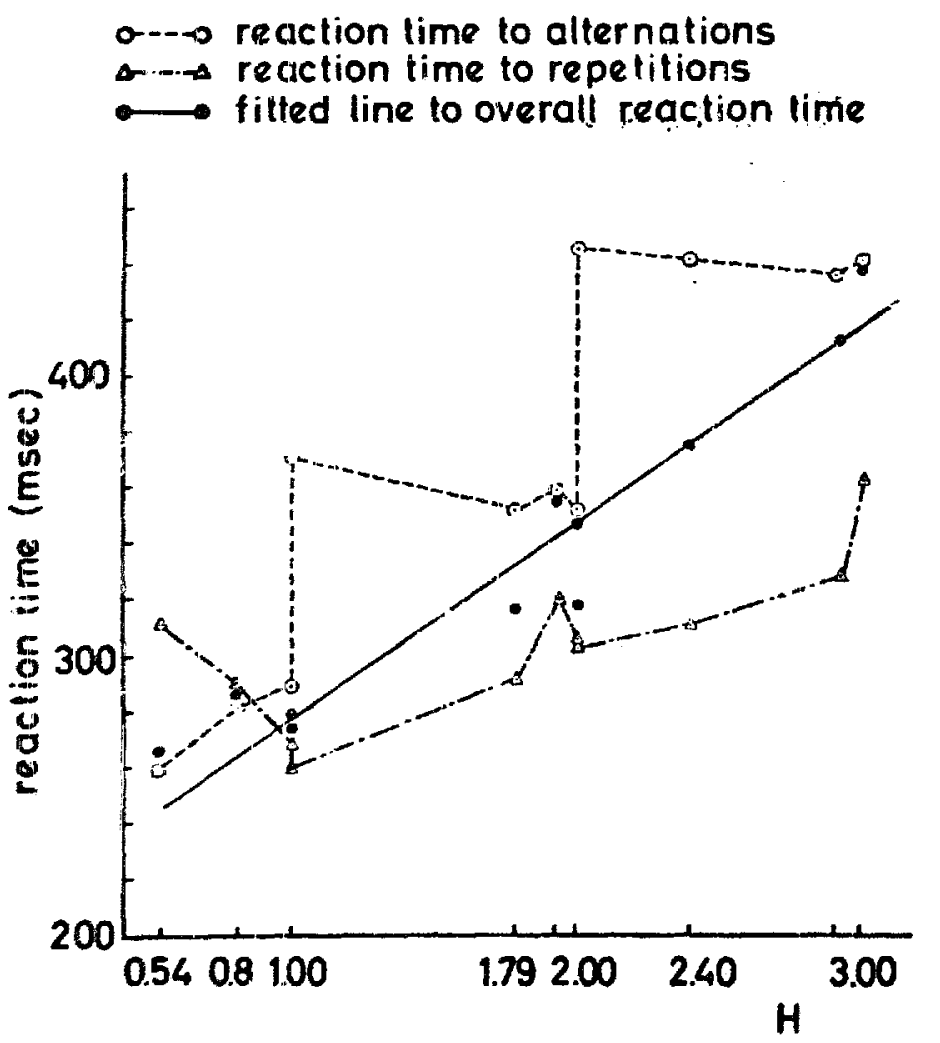

Fig. 1. The RT to repetitions, alternations, and the overall RT as a function of average stimulus urcertainty. The solid line has been fitted by eye to the $\bar{R} \bar{T}$ points. 
of ways, however, of particular interest to us now is that slice for which the probability of a signal is held constant at .5 and where $p_{\mathrm{a}}$ varies from .05 to .95. The data for the four subjects that were run on that slice thus far corroborate the findings of our main study. That is, $\bar{R} \bar{T}_{\mathrm{a}}$ and $\bar{R} \bar{T}_{\mathrm{r}}$ are sorale inverse function of $p_{\mathbf{a}}$ and $p_{\mathrm{r} .}$

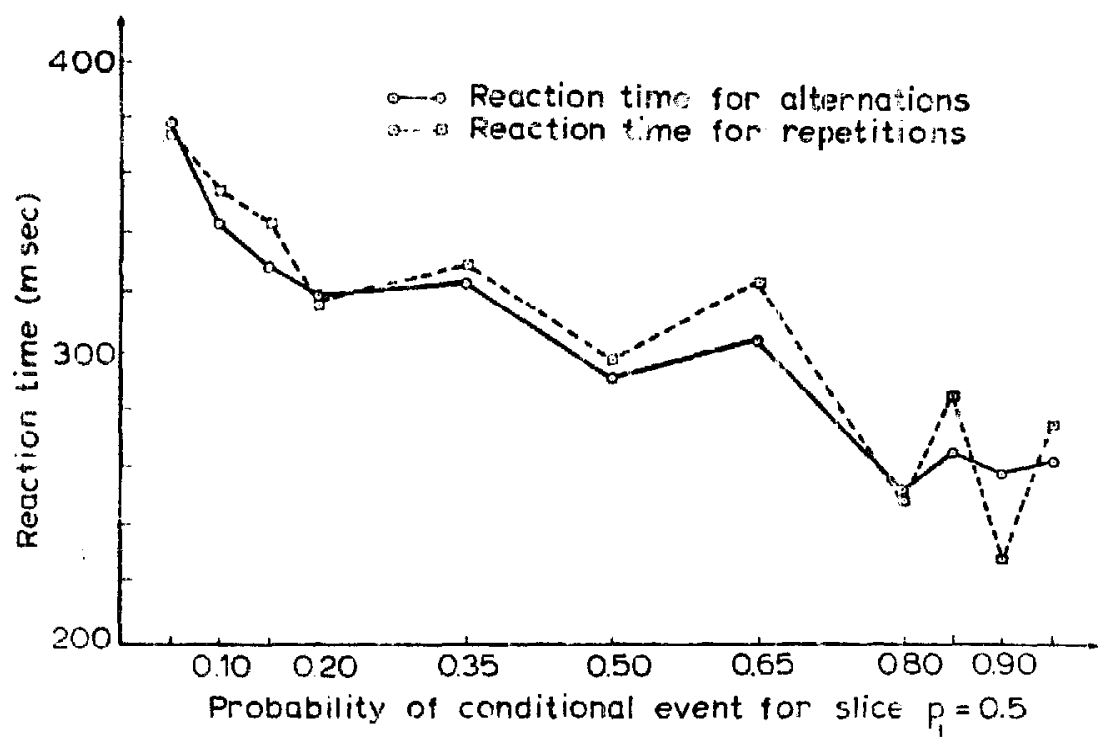

Fig. 2. Two-choice $R T$ for repetitions and alternations as a function of the probability of the conditional event, i.e. the probability of repetition and alternation respectively.

This relationship stands out quite clearly when the data are plotted as a function of the probability of the conditional event (see fig. 2). For example, $\bar{R} \bar{T}_{\mathrm{a}}$ for a sequence with $p_{\mathrm{a}}=.8$ is almost identical to $\bar{R} \bar{T}_{\mathrm{r}}$ for a sequence with $p_{\mathrm{a}}=.2$. In other words, in the equiprobable two-choice case $\bar{R} \bar{T}_{\mathrm{r}}$ and $\bar{R} T_{\text {a }}$ appear to be largely determined by the probability of the conditional event itself; the more frequent the event, the faster the time. Biccause of the fact that we used a $270 \mathrm{msec}$ delay in this experiment it becomes rather tenuous to ask whether an intrinsic difference between $\bar{R} \bar{T}_{\mathrm{r}}$ and $\bar{R} \bar{T}_{\mathrm{a}}$ remains to be accounted for, over and above the effects attributable to the probability of the events themselves. Such a difference has, of course, been found at $p_{2}=.5$ in our main study, as well as by Bertelson. What appears to have been established, however, is the fact that sume of the processes underlying performance in a two-choice task are quite different from those involving more than two choices.

Before returning to the main experiment, a simplified flow chart may wel! serve as a neuristic device for the remainder of the discussion (see fig. 3). This diagram is not to be interpreted as a model in any sense of the term. The main elements of the diagram are the two nodes: at the first node a decision must be made as to whether the present signal is a repetition or not. In the two choice case, of course, the process need not go any further for there is complete information at this point. However, for more than two-choice, 
if it is not a repetition, then the decision process goes on to the second node where a selection of the correct alternative must be made among the $(K-1)$ remaining alternatives. Since the number of remaining alternatives is always $(K-1)$ it would seem reasonable that if the time is determined by the number

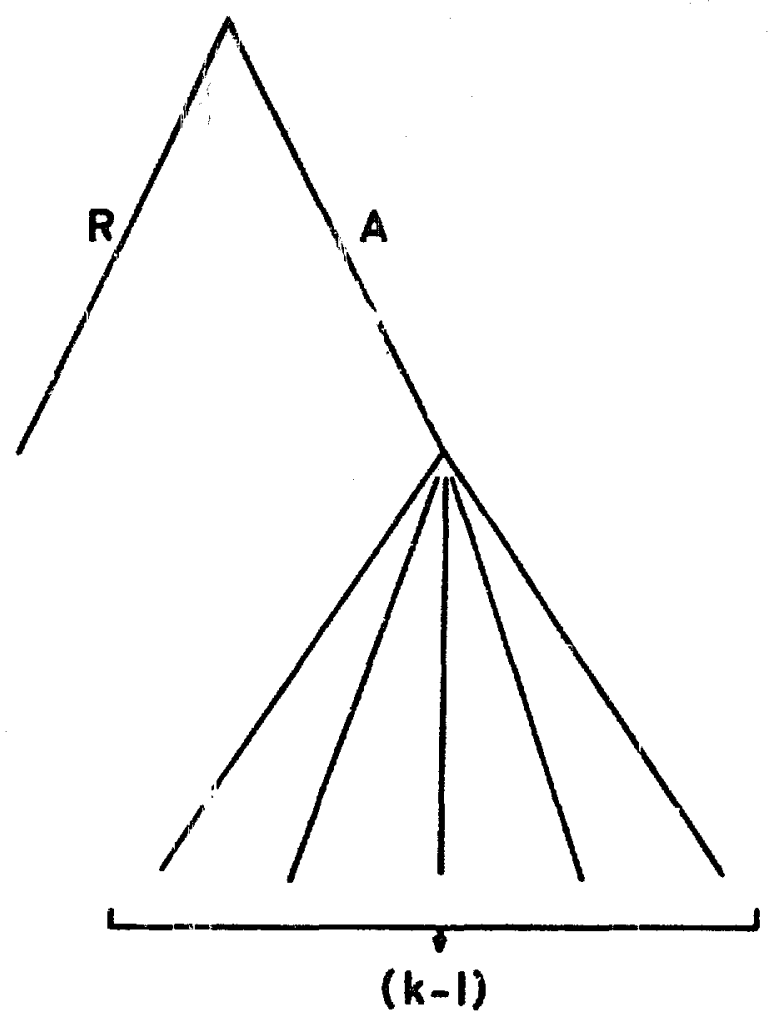

Fig. 3. Diagram of possible sequence of decisions involved in choice RT. At the first node, a decision is made as to whether the present signal is a repetition ( $R$ ) or an alternation (A); if it is an alternation, the next decision consists of a selection among, the $(K-1)$ remaining elements.

of items among which a selection must be made, $\bar{R} \bar{T}_{\mathrm{a}}$ should be constant for a given $K$, irrespective of the probability of alternations. However, the selection among these $(K-1)$ elements is not always correct-which leads me to my next poin: the error pattern for alternations and repetitions.

\section{ERror anALYSIS}

The overall error rate in this experiment was $2.7 \%$ and did not differ significantly inr the three days. Separating all the errors between those that occurred on repetition trials and those that occurred on alternation trials we note that over $94 \%$ of all errors, for the four and eight-choice sequences, were made on alternation trials. This error rate is, furthermore, completely insensitive to $p_{a}$. Once agairl, the two-choice case stands out as an exception to this (see table 2).

Let us next look at the proportion of alternation and repetition trials which resulted in errors and plot that proportion as a function of $H$ (ses fig. 4). 
TABle 2

Cell entries are the percent of all errors that occurred on alternation trials.

\begin{tabular}{cccccc}
$p_{a} \rightarrow$ & $1 / 2$ & $3 / 4$ & $7 / 8$ & $1 / 5$ & $3 / 8$ \\
$K$ & & & & & \\
\hline 2 & 82 & 59 & 33 & & \\
4 & 97 & 94 & 96 & 96 & \\
8 & 97 & 94 & 98 & & 94 \\
\hline
\end{tabular}

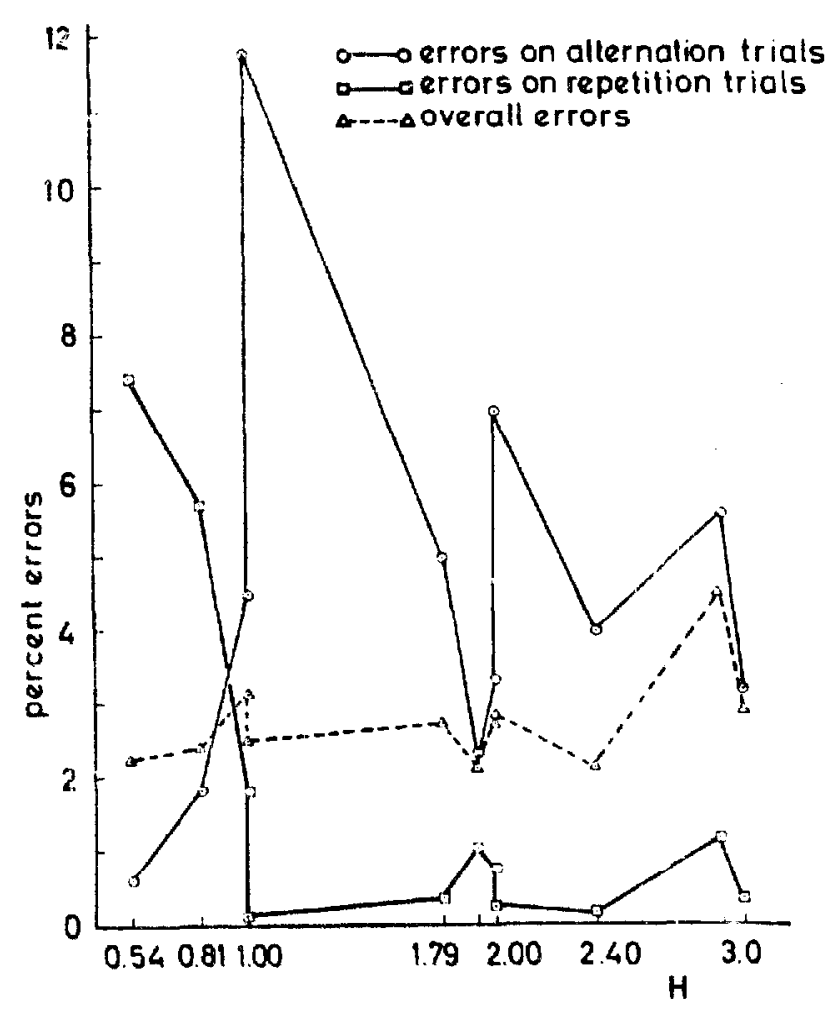

Fig. 4. Percent of alternation, repetition and overall trials on which errors occurred.

We note first, that for the four and eight-choice conditions less than $1 \%$ of the repetition trials resulted in errors, whereas the proportion of errors on alternation trials is consistently much higher. There also appears to be a systematic effect of $H$ and/or $p_{a}$ on the alternation trial errors which is not present in the errors made on the repetition trials. When the percent error on alternation trials is plotted against $p_{\mathrm{a}}$ (see fig. 5a), this systematic effect becomes quite apparent. In general, irrespective of $K$, the larger $p_{\mathrm{a}}$ the less the likelihood of an error on an aiternation trial. That is, subjects are increasingly prepared for non-repetitions the higher the probability of alternation in the sequence. 
The next question, of course, is huw well they are able to make such a preparation. In order to answer this question we turn to a detailed analysis of the types of errors that were malle on alternation trials. There are, of course, two ways that an error can be made on an alternation trial; either the subject repeats his previous response or he selects the incorrect non-repeating alternative; that is, he makes an erroneous alternation. Given that an error was made on an alternation trial, the a priori probability of the error being an erroneous alternation is $(K-2) /(K-1)$; that is .66 for the four-choice and .86 ror the eight-choice case. Plotting the percent of erroneous alternations as a function of $p_{2}$ (ste fig. $5 b$ ) we thote first that the percent of erroneous alternations is indeed higher for the eight-choice than for the four-choice conditions. Furthermore, the percent of such errors increases linearly as a function of $p_{\mathrm{a}}$. That is, as $p_{\mathrm{a}}$ goes uf the subject, in the course of increasing his state of readiness for a non-repetition, increases the likelihood that when an error does occur it will be an e roneous alternation.

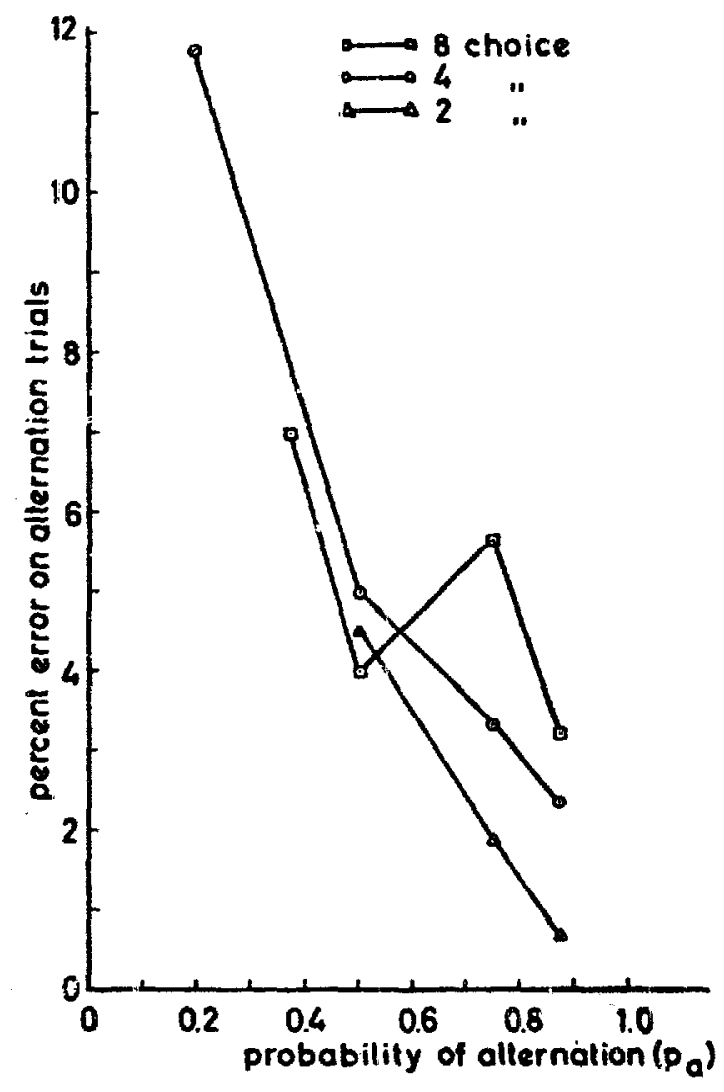

Fie 5a. Percent of aiternation trials on which errors occurred as a function of the probability of alternation for the two, four, and eight-choice conditions.

With this analysis of the error patterns the results appear to be quite clear. Both responses, the repetitions and the alternations, are sensitive to $p_{\mathrm{a}}$ as well as to $\boldsymbol{K}$. However, this only becomes apparent when errors in addition 


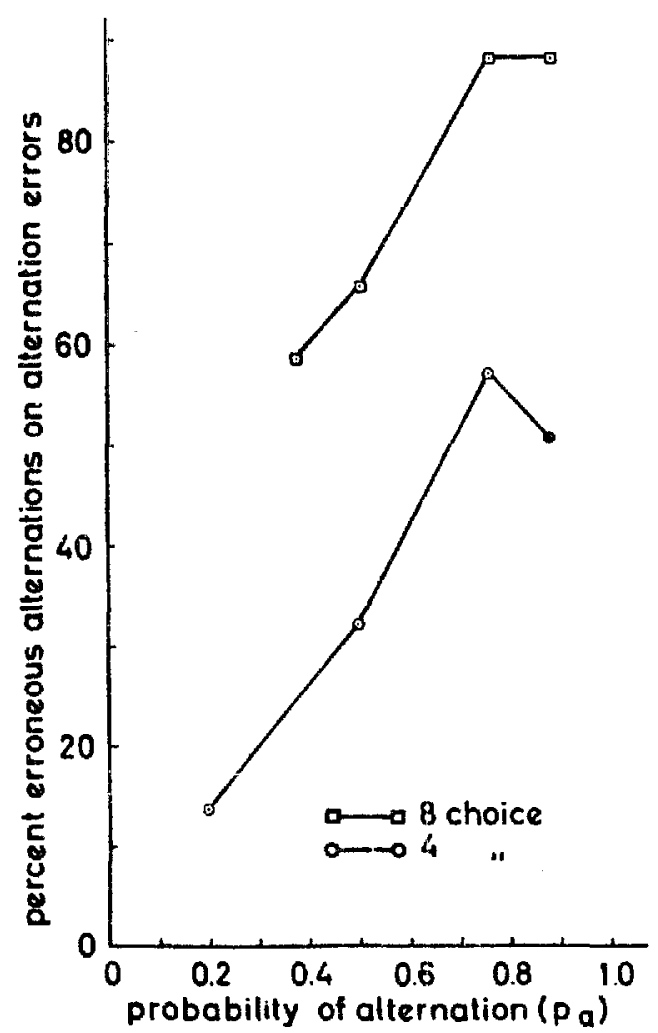

Fig. Sb. Given that an error occurred on an alternation trial, the percent of those errors consisting of erroneous alternations is plotted as a function of the probability of alternation for the four and eight-choice conditions.

to the reaction times are taken into account. Even though the errors constitute less than $3 \%$ of the total data they are quite systematic and are essential in providing a sense of coherence to the total picture.

\section{REFERENCES}

Bertelson, P., 1961. Quart. J. Psychol., 13, 90-102.

Bertelson, P., 1963. J. exp. Psychol., 655, 478-484.

Bertelson, P., 1965. Nature, 206, 217-218.

Hyman, R., 1953. J. Exp. Psychol., 45, 188-196.

Peretz, B. and S. Kornmblum, 1966. Med. and Biol. Engng., 4, 185-191.

Welford, A. T., 1959. Quart., J. exp. F'sychol., 11, 193-210. 